\title{
SPECTRAL ANALYSIS OF VIBRATION IN WEAKLY NON-LINEAR SYSTEMS
}

\author{
NGUYEN TIEN KHIEM \\ Institute of Mechanics, NCST of Vietnam
}

The weakly nonlinear systems subjected to deterministic excitations have been fully and deeply studied by use of the well developed asymptotic methods [14]. The systems excited by a random load have been investigated mostly using the Fokker-Plank-Kolmogorov equation technique combined with the asymptotic methods [5-8]. However, the last approach in most successful cases allows to obtain only a stationary single point probability density function, that contains no information about the correlation nor, consequently, the spectral structure of the response. The linearisation technique $[9,10]$ in general permits the spectral density of the response to be determined, but the spectral function obtained by this method because of the linearisation eliminates the effect of the nonlinearity. Thus, spectral structure of response of weakly nonlinear systems to random excitation, to the author's knowledge, has not been studied enough. This paper deals with the above mentioned problem. The main idea of this work is the use of an analytical simulation of random excitation given by its spectral density function and afterward application of the well known procedure of the asymptotic method to obtain an asymptotic expression of the response spectral density function. The obtained spectral relationship covers the linear system case and especially emphasizes the nonlinear effect on the spectral density of response. The theory will be illustrated by an example and at the end of this paper there will be a discussion about the obtained results.

\section{Simulation of stationary random process with given spectral den-} sity

Let's consider a stationary random process $X(t)$ with zero mean value and the spectral density function $S_{x}(\omega)$. This means that

$$
\langle X(t)\rangle=0 ; \quad\langle X(t) X(t+\tau)\rangle=R_{x}(\tau)=\int_{-\infty}^{+\infty} S_{x}(\omega) e^{-i \omega \tau} d \omega=2 \int_{0}^{\infty} S_{x}(\omega) \cos \omega \tau d \omega
$$




$$
S_{x}(\omega)=\frac{1}{2 \pi} \int_{-\infty}^{+\infty} R_{x}(\tau) e^{i \omega r} d \tau ; \quad \sigma_{x}^{2}=R_{x}(0)=\int_{-\infty}^{+\infty} S_{x}(\omega) d \omega=2 \int_{0}^{+\infty} S_{x}(\omega) d \omega
$$

The symbol $\langle$.$\rangle denotes the probability mean value operator and R_{x}(\tau)$ is the correlation function of the process $X(t)$.

Theorem. The given random process may be represented in the form

$$
X(t) \equiv X_{0}(t)=\sigma_{x} \sqrt{2} \cos (\Omega t+\varphi)
$$

where $\Omega$ is a random variable of the probability density: $P_{\Omega}(\omega)=2 S_{x}(\omega) / \sigma_{x}^{2}$; $\omega \in(0, \infty)$ and $\varphi$ is a random variable, uniformly distributed in $[0,2 \pi]$.

Proof. In fact

$$
\begin{aligned}
\left\langle X_{0}(t)\right\rangle & =\sigma_{x}\langle\sqrt{2} \cos \Omega t \cos \varphi-\sqrt{2} \sin \Omega t \sin \varphi\rangle \\
& =\sigma_{x} \sqrt{2}\langle\cos \Omega t\rangle\langle\cos \varphi\rangle-\sigma_{x} \sqrt{2}\langle\sin \Omega t\rangle\langle\sin \varphi\rangle=0
\end{aligned}
$$

because

$$
\langle\cos \varphi\rangle=\langle\sin \varphi\rangle=\frac{1}{2 \pi} \int_{0}^{2 \pi} \cos \varphi d \varphi=\frac{1}{2 \pi} \int_{0}^{2 \pi} \sin \varphi d \varphi=0 .
$$

Furthermore

$$
\begin{aligned}
\left\langle X_{0}(t) X_{0}(t+\tau)\right\rangle & =4 \int_{0}^{\infty} S_{x}(\omega) d \omega \frac{1}{2 \pi} \int_{0}^{2 \pi} \cos (\omega t+\varphi) \cos (\omega t+\varphi+\omega \tau) d \varphi \\
& =2 \int_{0}^{\infty} S_{x}(\omega) d \omega \frac{1}{2 \pi} \int_{0}^{2 \pi}[\cos (2 \omega t+\omega \tau+2 \varphi)+\cos \omega \tau] d \varphi \\
& =2 \int_{0}^{\infty} S_{x}(\omega) \cos \omega \tau d \omega=R_{x}(\tau) .
\end{aligned}
$$

So that $X_{0}(t)$ is a stationary random process with the correlation function $R_{x}(\tau)$ and consequently the spectral density $S_{x}(\omega)$. The theorem has been proved.

Since

$$
\cos (\Omega t+\varphi)=\frac{1}{2}\left[e^{i(\Omega t+\varphi)}+e^{-i(\Omega t+\varphi)}\right]
$$


the process $X(t)$ can be expressed in the complex form:

$$
X(t)=P_{0} e^{i \Omega t}+Q_{0} e^{-i \Omega t},
$$

where $P_{0}=\sigma_{x} \sqrt{2} e^{i \varphi} / 2, Q_{0}=P_{0}^{*}=\sigma_{x} \sqrt{2} e^{-i \varphi} / 2$. Thus, the given random process $X(t)$ with zero mean value and spectral density function $S_{x}(\omega)$ can be represented in the form (1.3).

\section{Asymptotic solution}

Now one shall consider stationary solution of the equation

$$
\ddot{y}(t)+2 h \dot{y}(t)+\omega_{0}^{2} y(t)+\varepsilon f(y, \dot{y})=X(t),
$$

here, $h, \omega_{0}, \varepsilon$ are constant parameters, $\varepsilon$ is a small one, $f$ is a non-linear function of $y, \dot{y}, X(t)$ - a stationary random process with zero mean value and spectral density function $S_{x}(\omega)$. The problem is to find the spectral density function of a stationary solution $y(t)$ of the equation (2.1). However, in this section an asymptotic solution of the equation (2.1) is constructed formally using the small parameter method.

Using expression (1.3) of the given process $X(t)$, the equation (2.1) can be rewritten in the form

$$
\ddot{y}(t)+2 h \dot{y}(t)+\omega_{0}^{2} y(t)+\varepsilon f(y, \dot{y})=P_{0} e^{i \omega t}+Q_{0} e^{-i \omega t},
$$

where $P_{0}=\sigma_{x} \sqrt{2} e^{i \varphi} / 2, Q_{0}=\sigma_{x} \sqrt{2} e^{-i \varphi} / 2, \Omega, \varphi$ are random processes defined in the previous section. Solution of the equation (2.2) is to be found now in the form

$$
y(t)=y_{0}(t)+\varepsilon y_{1}(t)+\varepsilon^{2} y_{2}(t)+\ldots
$$

Therefore, from (2.2) and (2.3) one get the equations for seeking the $y_{0}, y_{1}, y_{2}, \ldots$

$$
\begin{aligned}
& \ddot{y}_{0}(t)+2 h \dot{y}_{0}(t)+\omega_{0}^{2} y_{0}(t)=P_{0} e^{i \Omega t}+Q_{0} e^{-i \Omega t} \\
& \ddot{y}_{1}(t)+2 h \dot{y}_{1}(t)+\omega_{0}^{2} y_{1}(t)=-f\left(y_{0}, \dot{y}_{0}\right) \\
& \ddot{y}_{2}(t)+2 h \dot{y}_{2}(t)+\omega_{0}^{2} y_{2}(t)=-\frac{\partial f}{\partial y}\left(y_{0}, \dot{y}_{0}\right) y_{1}-\frac{\partial f}{\partial \dot{y}}\left(y_{0}, \dot{y}_{0}\right) \dot{y}_{1} .
\end{aligned}
$$

Solution of the equation (2.4) can be found in the form

$$
y_{0}(t)=A_{0} e^{i \Omega t}+B_{0} e^{-i \Omega t}
$$


where: $A_{0}=H(i \Omega) P_{0}, B_{0}=H(-i \Omega) Q_{0}, H(i \Omega)=\left[\omega_{0}^{2}-\Omega^{2}+2 h i \Omega\right]^{-1}$. This solution has another expression

$$
y_{0}(t)=\alpha(\Omega) e^{i \phi}+\alpha^{*}(\Omega) e^{-i \phi}
$$

with the following notations

$$
\alpha(\Omega)=\sigma_{x} \sqrt{2} H(i \Omega) / 2, \quad \Phi=\Omega t+\varphi, \quad \alpha^{*}(\Omega)=\sigma_{x} \sqrt{2} H(-i \Omega) / 2 .
$$

From (2.8) we will have

$$
\dot{y}_{0}(t)=\beta(\Omega) e^{i \phi}+\beta^{*}(\Omega) e^{-i \phi}, \quad \beta(\Omega)=i \Omega \alpha(\Omega) .
$$

Substituting (2.8) and (2.9) into the function $f(y, \dot{y})$ and, then, expanding it into the Fourier series yields

$$
f\left(y_{0}, \dot{y}_{0}\right)=\sum_{n} f_{n}(\Omega) e^{i n \phi}=\sum_{n} f_{n}(\Omega) e^{i n(\Omega t+\varphi)}
$$

with

$$
f_{n}(\Omega)=\frac{1}{2 \pi} \int_{0}^{2 \pi} f\left[y_{0}, \dot{y}_{0}\right] e^{-i n \phi} d \phi .
$$

The equation (2.5) together with expression (2.10) of the function $f(y, \dot{y})$, can be solved and its solution is of the form

$$
y_{1}(t)=\sum_{n} A_{n}(\Omega) e^{i n \phi}, \quad A_{n}(\Omega)=-H(i n \Omega) f_{n}(\Omega) .
$$

In this case, we will get

$$
\dot{y}_{1}(t)=\sum_{n} \bar{A}_{n}(\Omega) e^{i n \phi}, \quad \bar{A}_{n}(\Omega)=-i n \Omega H(i n \Omega) f_{n}(\Omega) .
$$

Substituting (2.8)-(2.9), (2.12)-(2.13) into the function

$$
g\left(y_{0}, \dot{y}_{0}, y_{1}, \dot{y}_{1}\right)=\frac{\partial f}{\partial y}\left(y_{0}, \dot{y}_{0}\right) y_{1}+\frac{\partial f}{\partial \dot{y}}\left(y, \dot{y}_{0}\right) \dot{y}_{1}
$$

makes the last a periodical function of $\phi$, which can consequently be expanded into the series

$$
g\left(y_{0}, \dot{y}_{0}, \dot{y}_{1}, \dot{y}_{1}\right)=\sum_{n} g_{n}(\Omega) e^{i n \phi}, \quad \phi=\Omega t+\varphi
$$


where

$$
g_{n}(\Omega)=\frac{1}{2 \pi} \int_{0}^{2 \pi} g\left[y_{0}, \dot{y}_{0}, y_{1}, \dot{y}_{1}\right] e^{-i n \phi} d \phi
$$

Substituting (2.14) for the function $g$ into the equation (2.6), one can get its solution as

$$
y_{2}(t)=\sum_{n} B_{n}(\Omega) e^{i n \phi} ; \quad B_{n}(\Omega)=-H(i n \Omega) g_{n}(\Omega)
$$

Thus, one has obtained formal expressions for $y_{0}(t), y_{1}(t), y_{2}(t)$.

3. Response spectral density function

Having the expressions of $y_{0}(t), y_{1}(t), y_{2}(t), \ldots$ in the form of $(2.8),(2.12)$, (2.16), one can go to the calculation of the correlation function

$$
\begin{aligned}
R_{y}(t, t+\tau)= & \left\langle y(t) y^{*}(t+\tau)\right\rangle \\
= & \left\langle y_{0}(t) y_{0}^{*}(t+\tau)\right\rangle+\varepsilon\left\langle y_{0}(t) y_{1}^{*}(t+\tau)+y_{0}(t+\tau) y_{1}^{*}(t)\right\rangle \\
& +\varepsilon^{2}\left\langle y_{0}(t) y_{2}^{*}(t+\tau)+y_{0}(t+\tau) y_{2}^{*}(t)+y_{1}(t) y_{1}^{*}(t+\tau)\right\rangle+\varepsilon^{3} \ldots \\
= & R_{0}(t, t+\tau)+\varepsilon R_{1}(t, t+\tau)+\varepsilon^{2} R_{2}(t, t+\tau)+\varepsilon^{3} \ldots
\end{aligned}
$$

At first, from (2.8) we have

$$
\begin{aligned}
R_{0}(t, t+\tau) & =\left\langle y_{0}(t) y_{0}^{*}(t+\tau)\right\rangle \\
& =\left\langle\left(\alpha e^{i \phi}+\alpha^{*} e^{-i \phi}\right)\left(\alpha^{*} e^{-i \phi} e^{-i \Omega t}+\alpha e^{i \phi} e^{i \Omega t}\right)\right\rangle \\
& =\left\langle\alpha \alpha^{*}\left(e^{-i \Omega t}+e^{i \Omega t}\right)\right\rangle+\left\langle\alpha^{2} e^{2 i \phi} e^{i \Omega t}+\alpha^{* 2} e^{-2 i \phi} e^{-i \Omega t}\right\rangle \\
& =2\left\langle\alpha \alpha^{*} \cos \Omega \tau\right\rangle+0 \\
& =\sigma_{x} \int_{0}^{\infty} P_{\Omega}(\omega)|H(i \omega)|^{2} \cos \omega \tau d \omega \\
& =2 \int_{0}^{\infty}|H(i \omega)|^{2} S_{x}(\omega) \cos \omega \tau d \tau \\
& =R_{0}(\tau) .
\end{aligned}
$$

Furthermore, using (2.8) and (2.12), function $R_{1}(t, t+\tau)$ takes the form 


$$
\begin{aligned}
R_{1}(t, t+\tau)= & \left\langle y_{0}(t) y_{1}^{*}(t+\tau)+y_{0}^{*}(t+\tau) y_{1}(t)\right\rangle \\
= & \left\langle\left(\alpha e^{i \phi}+\alpha^{*} e^{-i \phi}\right) \sum_{n} A_{n}^{*} e^{-i n(\phi+\Omega \tau)}\right\rangle \\
& +\left\langle\left(\alpha^{*} e^{-i \phi} e^{-i \omega t}+\alpha e^{i \phi} e^{i \omega t}\right) \sum_{n} A_{n} e^{i n \phi}\right\rangle \\
= & \left\langle\alpha A_{1}^{*} e^{-i \Omega t}+\alpha^{*} A_{-1}^{*} e^{i \Omega t}\right\rangle+\left\langle\alpha^{*} A_{1} e^{-i \Omega t}+\alpha A_{1} e^{i \omega t}\right\rangle \\
= & 2\left\langle\left(\alpha A_{1}^{*}+\alpha^{*} A_{1}\right) \cos \Omega \tau\right\rangle \\
= & -\left(4 \sqrt{2} / \sigma_{x}\right) \int_{0}^{\infty} S_{x}(\omega)|H(i \omega)|^{2} \operatorname{Re} f_{1}(\omega) \cos \omega \tau d \omega \\
= & R_{1}(\tau) .
\end{aligned}
$$

Finally, the function

$$
R_{2}(t, t+\tau)=\left\langle y_{0}(t) y_{2}^{*}(t+\tau)+y_{0}(t+\tau) y_{2}^{*}(t)+y_{1}(y) y_{1}^{*}(t+\tau)\right\rangle
$$

can be determined as follows. First note that the series (2.12) is different to series (2.16) by the coefficients $A_{n}$ and $B_{n}$, and therefore, by the functions $f_{n}, g_{n}$. So that the function.

$$
R_{20}(t, t+\tau)=\left\langle y_{0}(t) y_{2}^{*}(t+\tau)+y_{0}(t+\tau) y_{2}^{*}(t)\right\rangle
$$

could be obtained from the $R_{1}(\tau)$ defined in (3.2) by putting the $g_{1}(\omega)$ instead of the $f_{1}(\omega)$. As a result

$$
R_{20}(t, t+\tau)=-\left(4 \sqrt{2} / \sigma_{x}\right) \int_{0}^{\infty} S_{x}(\omega)|H(i \omega)|^{2} \operatorname{Re} g_{1}(\omega) \cos \omega r d \omega=R_{20}(\tau)
$$

To finish determining $R_{2}$, it remains to calculate the correlation function of the process $y_{1}(t)$ :

$$
\begin{aligned}
R_{21}(t, t+\tau) & =\left\langle y_{1}(t) y_{1}^{*}(t+\tau)\right\rangle \\
& =\sum_{n, m}\left\langle A_{n}(\Omega) A_{m}(\Omega) e^{i(n-m) \phi-i m \Omega \tau}\right\rangle \\
& =\sum_{n}\left\langle A_{n}(\Omega) A_{n}^{*}(\Omega) e^{-i n \Omega \tau}\right\rangle \\
& =\sum_{n}\left\langle\left|H(i n \Omega) f_{n}(\Omega)\right|^{2} e^{-i n \Omega \tau}\right\rangle \\
& =4 \sum_{n=1}^{\infty} \int_{0}^{\infty}|H(i n \omega)|^{2}\left|f_{n}(\omega)\right|^{2} S_{x}(\omega) \cos n \omega \tau d \omega \\
& =R_{21}(\tau) .
\end{aligned}
$$


Thus, the correlation function $R_{y}$ of the solution (2.3) gets the final form

$$
R_{y}(t, t+\tau)=R_{0}(\tau)+\varepsilon R_{1}(\tau)+\varepsilon^{2} R_{20}(\tau)+\varepsilon^{2} R_{21}(\tau)+\varepsilon^{3} \cdots=R_{y}(\tau),
$$

where the functions $R_{0}(\tau), R_{1}(\tau), R_{20}(\tau), R_{21}(\tau)$ have just been determined above. The obtained expression of the correlation function shows that the asymptotic solution (2.3) constructed above is really a stationary random process. So that its spectral density can be found after performing the Fourier transform of the correlation function (3.4):

$$
S_{y}(\omega)=J\left\{R_{y}(\tau)\right\}=S_{0}(\omega)+\varepsilon S_{1}(\omega)+\varepsilon^{2} S_{20}(\omega)+\varepsilon^{2} S_{21}(\omega)+\varepsilon^{3} \ldots
$$

where

$$
\begin{aligned}
S_{0}(\omega) & =J\left\{R_{0}(\tau)\right\}=|H(i \omega)|^{2} S_{x}(\omega) ; H(i \omega)=\left[\omega_{0}^{2}-\omega^{2}+i h \omega\right]^{-1}, \\
S_{1}(\omega) & =J\left\{R_{1}(\tau)\right\}=-\frac{2 \sqrt{2}}{\sigma_{x}}|H(i \omega)|^{2} \operatorname{Re} f_{1}(\omega) S_{x}(\omega), \\
S_{20}(\omega) & =J\left\{R_{20}(\tau)\right\}=-\frac{2 \sqrt{2}}{\sigma_{x}} \mid H\left(\left.i(\omega)\right|^{2} \operatorname{Re} g_{1}(\omega) S_{x}(\omega)\right. \\
S_{21}(\omega) & =J\left\{R_{21}(\tau)\right\}=2|H(i \omega)|^{2} \sum_{n=1}^{\infty} \frac{1}{n}\left|f_{n}(\omega / n)\right| S_{x}(\omega / n)
\end{aligned}
$$

The effect of nonlinearity would be more obvious in formula (3.5) rewritten in the form

$$
S_{y}(\omega)=|H(i \omega)|^{2}\{1+\mu(\omega)\} S_{x}(\omega),
$$

where coefficient $\mu(\omega)$ describing the non-linear effect is

$$
\mu(\omega)=-\frac{2 \sqrt{2}}{\sigma_{x}}\left[\varepsilon \operatorname{Re} f_{1}(\omega)+\varepsilon^{2} \operatorname{Re} g_{1}(\omega)\right]+2 \varepsilon^{2} \sum_{n=1}^{\infty} \frac{1}{n}\left|f_{n}(\omega / n)\right|^{2} \frac{S_{x}(\omega / n)}{S_{x}(\omega)}+\varepsilon^{3} \ldots
$$

In formula (3.11) one can see also the contribution of the so-called subharmonic oscillation to the response spectral structure of the system. Furthermore, since the spectral density of a stationary random process is always positive, the parameters $\varepsilon, h, \sigma_{x}, \omega_{0}$. must satisfy the condition

$$
D(\omega)=1+\mu(\omega) \geq 0, \quad \forall \omega .
$$

It is also the condition for the existence of the stable stationary solution of the given system (2.1). 


\section{Results and discussion}

To illustrate the theory, in this section the system

$$
\ddot{y}(t)+2 h \dot{y}(t)+y(t)+\varepsilon y^{3}(t)=X(t)
$$

is considered under the assumption that the excitation $X(t)$ is a finite white noise process, which has the spectral density

$$
S_{x}(\omega)=\left\{\begin{array}{lll}
0.25 & \text { for } & \omega \in(-2,+2) \\
0 & & \text { elsewhere }
\end{array}\right.
$$

For such a process $\sigma_{x}=1$.

In this case one has

$$
\begin{aligned}
& f_{1}(\omega)=\frac{3 \sqrt{2}}{4}|H(i \omega)|^{2} H(i \omega) ; \\
& f_{3}(\omega)=\frac{\sqrt{2}}{4} H^{3}(i \omega) \\
& H(i \omega)=\left(1-\omega^{2}+2 i h \omega\right)^{-1} \\
& g_{1}(\omega)=-\frac{3 \sqrt{2}}{8}|H(i \omega)|^{4} H(i \omega)\{6 H(i \omega)+3 H(-i \omega)+H(3 i \omega)\} .
\end{aligned}
$$

Therefore,

$$
\begin{aligned}
\mu(\omega)= & -3 \varepsilon|H(i \omega)|^{2} \operatorname{Re}\{H(i \omega)\}+\frac{\varepsilon^{2}}{12}|H(i \omega / 3)|^{6}+\frac{27 \varepsilon^{2}}{4}|H(i \omega)|^{6} \\
& +\frac{3 \varepsilon^{2}}{2}|H(i \omega)|^{4} \operatorname{Re}\{H(i \omega)[6 H(i \omega)+H(3 i \omega)]\},
\end{aligned}
$$

and

$$
S_{y}(\omega)=\frac{1}{4}|H(i \omega)|^{2}\{1+\mu(\omega)\}, \quad H(i \omega)=\left[1-\omega^{2}+2 i h \omega\right]^{-1}, \quad \omega \in[0,2]
$$

Graphics of the functions $S_{y}(\omega), \omega \in[0,2]$ for various values of the parameters $h$, $\varepsilon$ are plotted and shown in figures 1-3. Comparing the figures 1,2 one can see clearly the effect of the nonlinearity on the response spectrum. It's namely, that the one peak spectral curve, typical for linear systems, now becomes a two peak curve in the presence of nonlinearity. The new peaks in the spectral curve are more obvious and sharp, as the magnitude of the nonlinearity increases and the damping is reduced. 


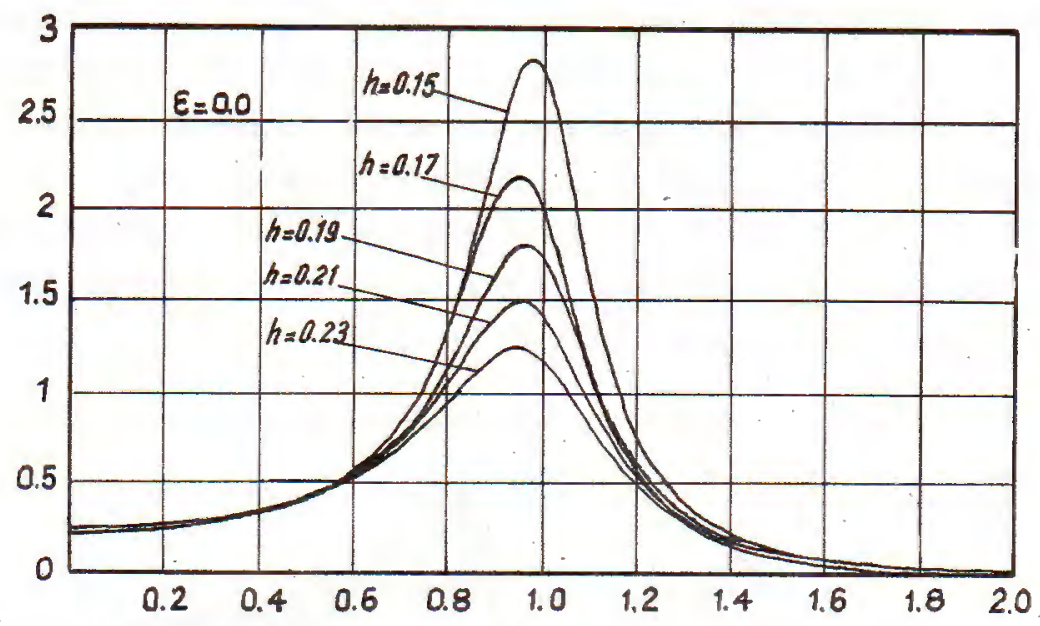

Fig. 1

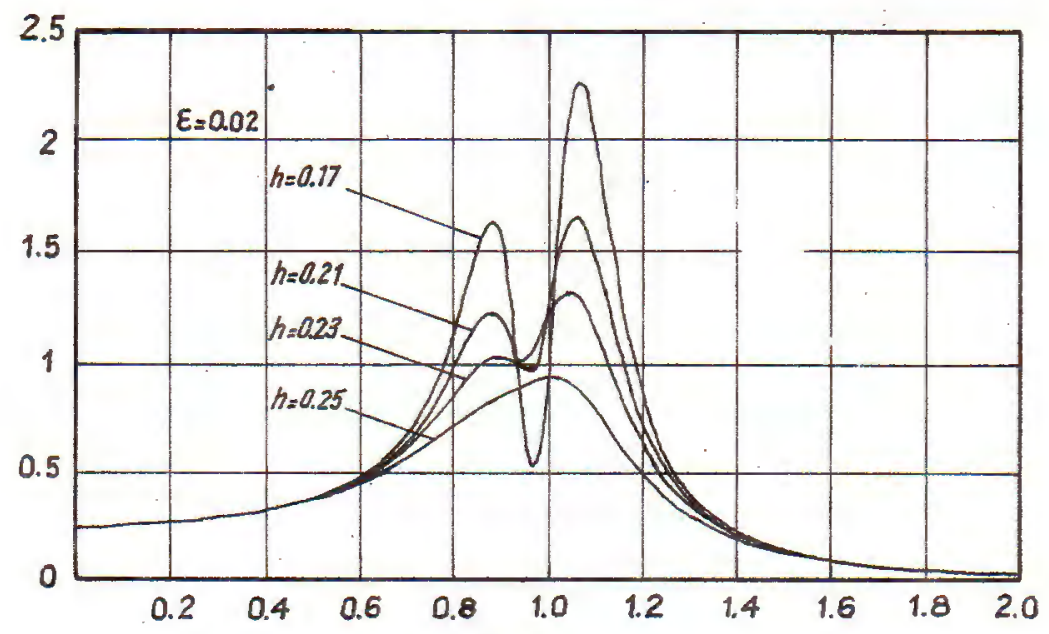

Fig. 2

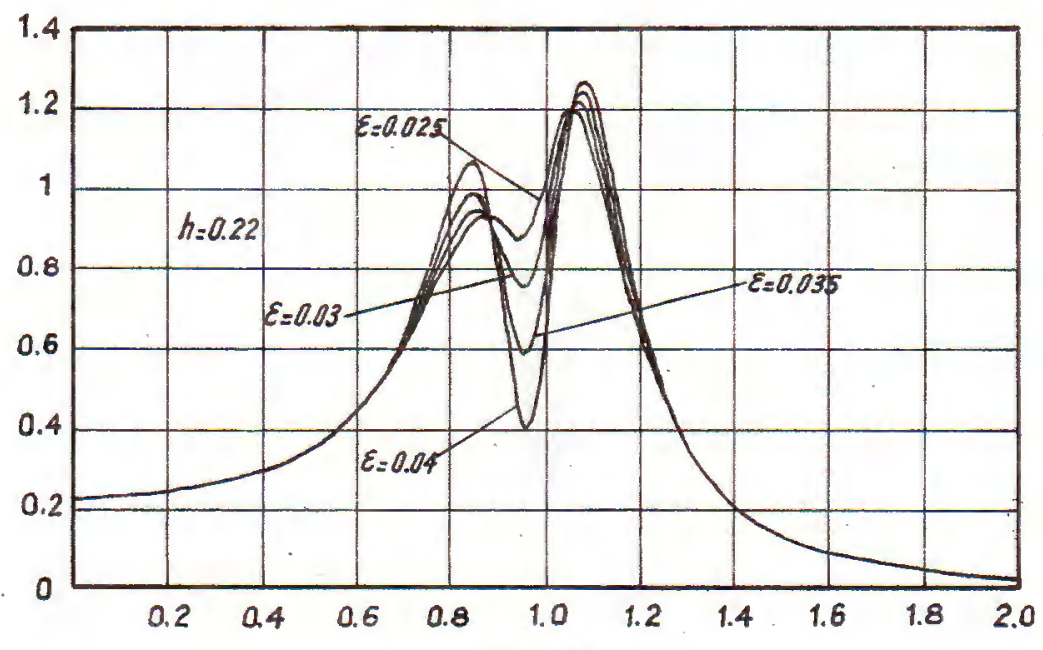

Fig. 3 
Graphics of the functions $D(\omega)=1+\mu(\omega), \omega \in[0,2]$ in two cases: $h=0.2$, $\varepsilon=0.028-0.038$ and $h=0.3, \varepsilon=0.08-0.12$ are plotted in figures 4,5 . These figures show an interesting fact that for the given damping ratio $h$ there exist exciting frequencies, at which the amplitude of excited oscillation is not affected by the nonlinearity. The condition for the existence of stable stationary oscillation (3.12) in this case may be determined only numerically and in the plane of $(h, \varepsilon)$ the condition defines the unshaded zone in figure 6.

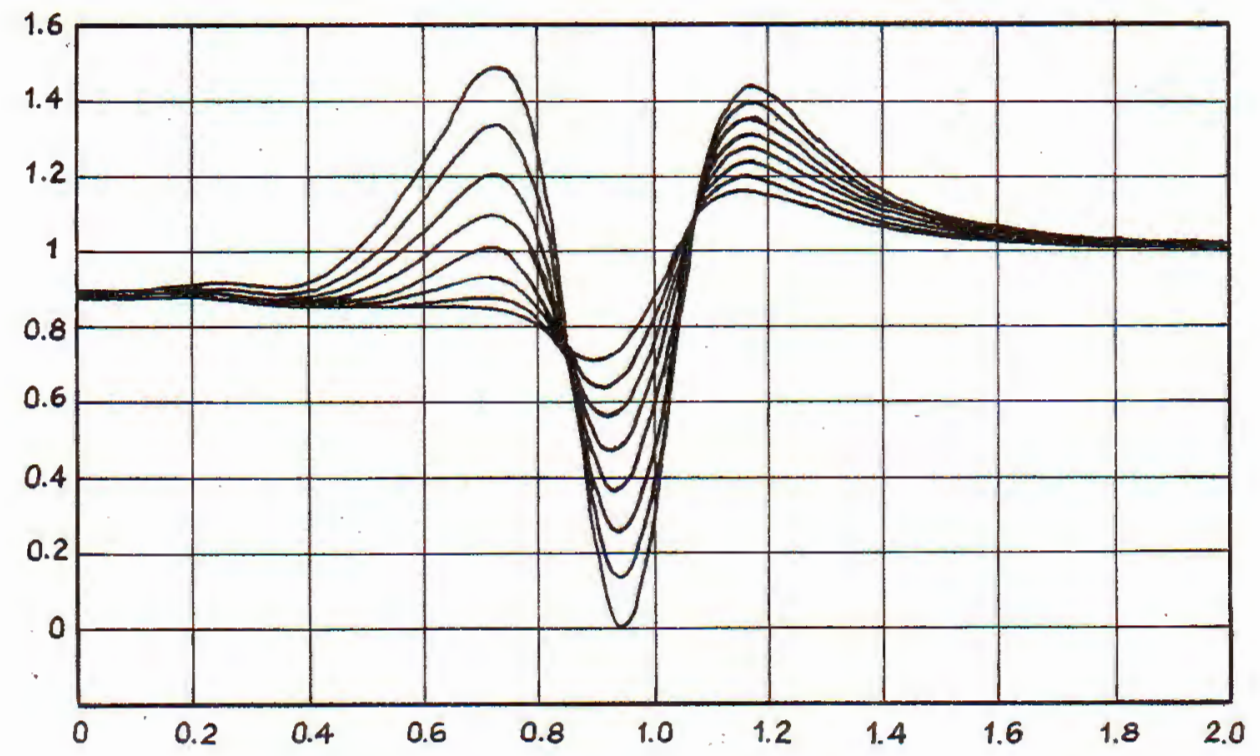

Fig. 4. Function $D(\omega)$ for $h=0.2 ; \varepsilon=0.028 ; 0.002 ; 0.038$

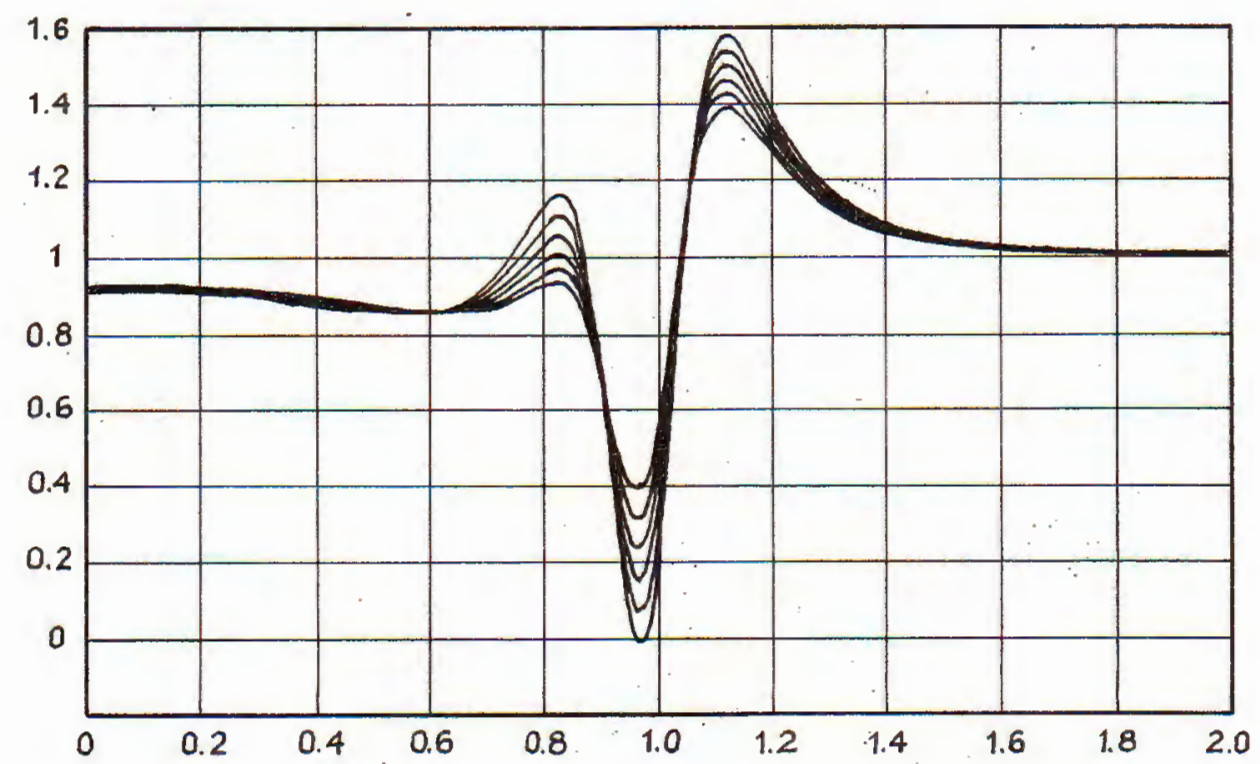

Fig. 5. Function $D(\omega)$ for $h=0.3 ; \varepsilon=0.08 ; 0.002 ; 0.12$ 


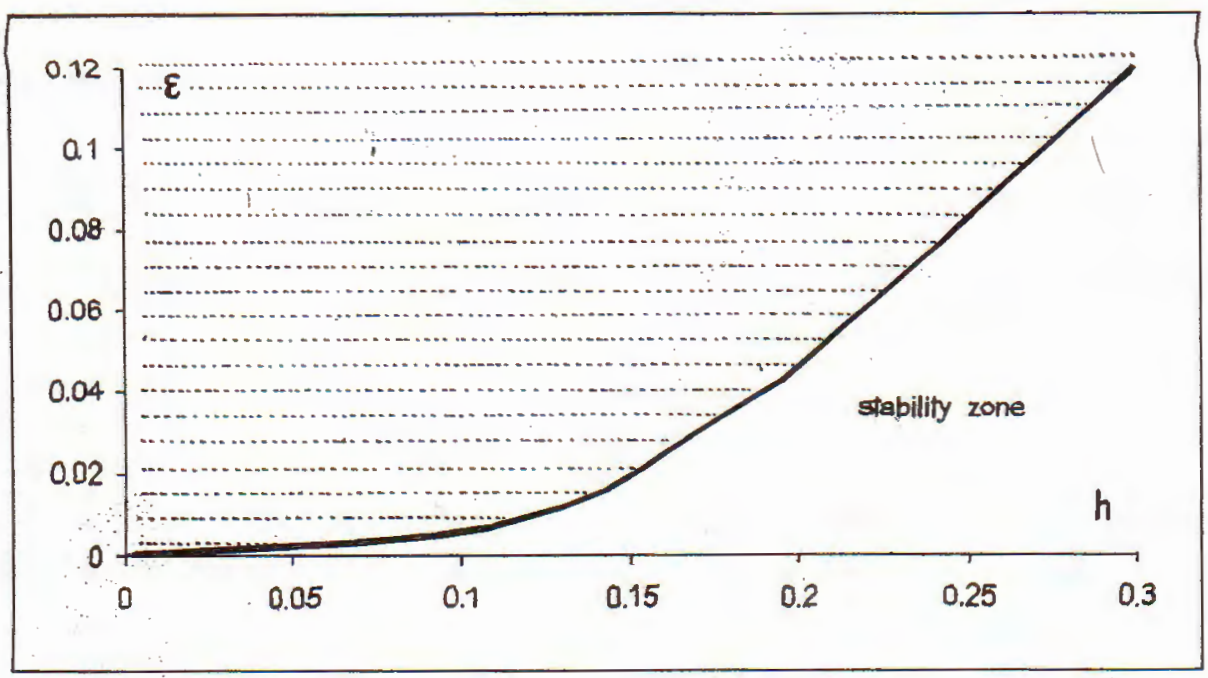

Fig. 6. Zone for existence of stable stationary solution

\section{Conclusion}

Based on the obtained results, the following conclusions can be given:

1. Weakly nonlinear systems subjected to a stationary random excitation have been considered.

2. Using a harmonic simulation of a stationary random process with the given spectral density and the procedure of the well known small parameter method, an asymptotic expression for spectral density of response of the systems is established.

3. The response spectral density function obtained without any assumption about the smallness of the damping and excitation clearly contains the nonlinear effect, that is illustrated in presented example by the presence of two peaks in the spectral density function of response.

4. The obtained formula for response spectral density function has also shown the effect of subharmonic oscillation.

5. From the obtained formula, a condition for the existence of stationary solution of the systems can be obtained.

6. The approach proposed herein may be further developed to investigate other cases of weakly nonlinear systems.

This work has been completed with financial support from The National Council for Natural Sciences of Vietnam. 


\section{REFERENCES}

1. Bogoliubov N. N., Mitropolskii Yu. A. Asymptotic methods in the theory of nonlinear oscillations. Moscow 1974.

2. Kauderer H. Nichtlineare mechanik. Springer-Verlag, Berlin 1958.

3. Mitroplolskii Yu. A., Nguyen Van Dao. Applied asymptotic methods in nonlinear oscillations. Kluwer Academic Publishers, 1997.

4. Nguyen Van Dao, Nguyen Van Dinh. Interactions between nonlinear oscillating systems. VNU Publishing House, Hanoi 1999.

5. Mitropolskii Yu. A., Kolomiets V. G. Application of the asymptotic methods in stochastic systems. In "Approximate methods applied to nonlinear systems", Kiew 1976.

6. Bolotin V. V. Random vibration of elastic systems. Moscow 1979.

7. Nguyen Dong Anh. Effect of combined random and periodic excitations on the nonlinear vibratory systems. D. Sc. Thesis, Kiev 1986.

8. Nguyen Tien Khiem. Application of the Fokker-Planck-Kolmogorov equation - method to study random vibrations of systems with delay. D.Sc. Thesis, Kiev 1992.

9. Crandall S. H. Heurestic and equivalent linearization techniques for random vibration of nonlinear oscillators. In "Proceedings of the $8^{\text {th }}$ International Conference on Nonlinear Oscillation", Prague 1978.

10. Roberts J. B. and Spanos P. D. Random vibration and statistical linearization. Wiley, N. Y. 1990.

Received July 6, 2000

\section{PHÂN TÍCH PHỔ DAO ĐộNG TRONG HỆ PHI TUYẾN YẾU}

Trên cơ sở mô phỏng điều hòa tác động ngẫu nhiên dừng và khai triển nghiệm theo chuỗi tiệm cận của tham số bé đã xây dựng được công thức tiệm cận cưa hàm mật độ phổ cho nghiệm hệ phi tuyến yếu. Ở đây lực cản và kích động ngoài không cần phải giả thiết nhỏ cùng bậc với thành phần phi tuyến. Công thức này cho phép nghiên cứu ảnh hưởng rô rệt của yếu tố phi tuyến và dao động thứ điều hòa đến đặc trưng phổ của dao động. Trong ví dụ đã xét hệ Duffing dưới tác động của ồn trắng dải hẹp. Kết quả cho thấy thành phần phi tuyến đã làm cho hàm phổ một đỉnh đặc trưng của hệ tuyến tính trở thành hàm có hai đỉnh rõ rệt. Phương pháp này có thể phát triển tiếp để nghiên cứu các hệ tự dao động hoặc các hệ kích động tham số. 